\title{
Simulations of model magnetorheological fluids in squeeze flow mode
}

Article

Accepted Version

Ruiz-López, J. A., Wang, Z. W., Hidalgo-Álvarez, R. and de Vicente, J. (2017) Simulations of model magnetorheological fluids in squeeze flow mode. Journal of Rheology, 61 (5). pp. 871-881. ISSN 0148-6055 doi: https://doi.org/10.1122/1.4990641 Available at https://centaur.reading.ac.uk/70790/

It is advisable to refer to the publisher's version if you intend to cite from the work. See Guidance on citing.

To link to this article DOI: http://dx.doi.org/10.1122/1.4990641

Publisher: Society of Rheology

All outputs in CentAUR are protected by Intellectual Property Rights law, including copyright law. Copyright and IPR is retained by the creators or other copyright holders. Terms and conditions for use of this material are defined in the End User Agreement.

\section{www.reading.ac.uk/centaur}

\section{CentAUR}

Central Archive at the University of Reading 
Reading's research outputs online 


\title{
Simulations of model magnetorheological fluids in squeeze flow mode
}

\author{
J. A. Ruiz-López, ${ }^{1}$ Z. W. Wang, ${ }^{2}$ \\ R. Hidalgo-Álvarez ${ }^{1}$ and J. de Vicente ${ }^{1}$ \\ ${ }^{1}$ Department of Applied Physics, Faculty of Sciences, University of Granada, 18071- \\ Granada, Spain - jvicente@ugr.es \\ ${ }^{2}$ School of Mathematical, Physical and Computational Sciences, University of \\ Reading, Reading RG6 6AX, UK - zuowei.wang@reading.ac.uk
}

\section{Submitted to Journal of Rheology}

\begin{abstract}
A particle-level simulation methodology is proposed to study the squeeze flow behavior of model magnetorheological (MR) fluids. The simulation algorithm takes into account Brownian motion and local field corrections to magnetic interactions of the particles. Simulation results obtained from using different initial configurations, including one single-particle-width chain per simulation box, random or lattice arrangements of preassembled single-particle-width chains as well as randomly dispersed particle suspensions, are compared with experimental data and predictions of a recently developed microscopic model. The assumption of single-particle-width chain structures in the systems has been shown to generate normal stresses larger than those found in experiments and the micromechanical model. However, much better agreement between the simulation and experimental results have been reached when using random initial configurations in the simulations.
\end{abstract}

\section{Keywords}

Magnetorheology, magnetorheological fluids, yield stress, squeeze flow, computer simulation. 


\section{INTRODUCTION}

Magnetorheological (MR) fluids are typically suspensions of magnetizable particles in a liquid carrier. In the presence of external magnetic fields, the particles aggregate forming anisotropic structures as a result of interparticle magnetostatic interactions. These structures are behind a rich rheological behavior that spans from a liquid-like system at low fields to a solid-like paste, exhibiting an apparent yield stress, in the presence of sufficiently large fields [1-3].

Currently, there exists an important portfolio of devices incorporating the MR technology, most of them related to stress transfer control. In these devices, MR fluids are subject to very complex flows. However, most of the studies reported in the literature concern viscometric flows, and in particular, steady shear flow tests. Very few works have been done involving other kinds of flows (e. g., elongational flows) in spite of the fact that recent investigations on squeeze (i. e., compression) flows demonstrate a significantly larger yield stress than in conventional shearing flows [4-6]. Undoubtedly, the rheological behavior of MR fluids under compression is not completely understood yet.

During the last decade, several experimental studies have been reported under constant volume operation, creeping flow approximation and for low plasticity numbers (slow speeds) [5,7-9]. In these works, experimental data are found to match reasonably well with predictions from continuous media theories in Bingham plastics. These theories lead to a yield compressive stress $\tau_{Y C}$ that is related to the usual yield (shear)

stress $\tau_{y}$ as follows: $\tau_{Y C}=\frac{2}{3} \sqrt{\frac{V}{\pi h_{0}{ }^{3}}} \tau_{y}$, where $V$ is the constant volume and $h_{0}$ is the initial gap. Here, it is important to note that throughout the current work the notation "yield stress" refers to the "apparent yield stress". Therefore, the dependence of the yield compressive stress on the magnetic field strength and the particle volume fraction is solely provided by the dependence of the yield (shear) stress on the magnitudes of these two quantities. It is also important to note that if the initial gap is small compared to the initial radius of the sample, $h_{0} \ll r_{0} \sim \sqrt{V / h_{0}}$, the yield compressive stress can be much higher than the yield (shear) stress. 
Interestingly, previous works demonstrated that the yield (shear) stress increases during the compression stage due to the so-called strengthening effect [10]. This was claimed to be so because chain-like structures become thicker under compression [11]. The strengthening effect has been theoretically explained using the local field theory by Zhang et al. [12], and more recently interpreted in terms of the Péclet number [13]

In a recent study, Ruiz-López et al. [14] developed a micromechanical model to explain the rheological properties of MR fluids under slow compression. They found that the yield compressive stress depends linearly on the particle volume fraction and on the magnetic field strength squared: $\tau_{Y C} \sim \phi H^{2}$. The validity of this model is limited to dilute MR fluids at sufficiently small deformations and therefore some deviations from experimental results were found at the particle volume fractions used in commercial applications. To get a better insight, in the current work we aim to use particle-level simulations to explore the yielding behavior of MR fluids under compression flows.

Simulations of field-responsive fluids under compression are very scarce in the literature. Lukkarinen and Kaski [15-16] and Kim et al. [17] carried out simulations of electrorheological (ER) fluids. In these works the compressive stress sharply increases as a function of the compressive strain and the yield compressive stress is higher than the yield (shear) stress (in accordance with available experimental data). However, these simulations were performed at a constant voltage and consequently the electric field strength increased under compression, complicating the analysis. To the best of our knowledge there is only one published work dealing with the simulation of MR fluids under compression [5]. In that particular study, the external magnetic field strength remains constant during compression, facilitating the interpretation of the results. The authors reported particle-level molecular dynamic simulations and these data satisfactorily matched with experimental results. However, the number of particles in those simulations was limited due to the non-periodical boundary conditions and results were only obtained at a particle volume fraction of $5 \mathrm{vol} \%$.

In this paper we propose a novel particle-level simulation methodology to explore the squeeze flow behavior of MR fluids using periodic boundary conditions (PBCs) and local field corrections. We pay special attention to the influence of particle microstructures on the yield compressive stress as a function of the particle volume 
fraction. Simulation results are compared with experimental data, predictions of continuous media theories and micromechanical models available in the literature $[5,14]$.

\section{SIMULATION MODEL}

Brownian dynamics simulations were carried out to study the rheological behavior of model MR fluids under slow compression. The MR fluid was modeled as a suspension of $N$ monodisperse buoyant and inertialess particles with radius $a$ dispersed in a Newtonian fluid. The volume $V$ of the rectangular simulation box is a function of the number of particles $N$ and the particle volume fraction $\phi$ as follows: $V=$ $4 \pi a^{3} N / 3 \phi$. We chose a Cartesian coordinate system centered in the simulation box as shown in the Figure 1.

During the compression test, the two horizontal plates approached each other at a constant relative velocity, namely, the upper plate moved down with a velocity of $-v / 2 \hat{z}$ while the lower plate moved up with a velocity of $+v / 2 \hat{z}$. Here $\hat{z}$ is the unit vector in the $z$-direction. PBCs were applied in the transversal directions ( $x$ - and $y$ directions). Sticky or no-slip boundary conditions were applied for particles within a distance of $1.1 a$ to the surfaces of the walls, which effectively prevent the chain- or column-like structures from sliding along the walls in the transverse directions. This may potentially lead to an increase in the local concentration of particles around the gap-spanning structures during compression. But particles not in close proximity of the walls can still move in the $\mathrm{x}$ - and $\mathrm{y}$-directions due to Brownian motion and the squeeze flow. The volume of the simulation box was conserved in all simulation runs. This means that during the compression, the lateral box length $l(t)$ increases, while the gap between the plates $h(t)$ diminishes according to $l(t)=\sqrt{V / h(t)}$.

For simplicity, magnetostatic interaction was modeled using the point-dipole approximation. Hence, the magnetostatic force between two particles, $i$ and $j$, separated by a distance, $r_{i j}$, is given by:

$$
\vec{F}_{i j}{ }^{m a g}=\frac{3}{4 \pi \mu_{0} \mu_{c r}} \frac{\left[3\left(\vec{m}_{i} \cdot \hat{r}\right)\left(\vec{m}_{j} \cdot \hat{r}\right)-\vec{m}_{i} \cdot \vec{m}_{j}\right] \hat{r}-\left[\left(\vec{m}_{i} \cdot \widehat{\theta}\right)\left(\vec{m}_{j} \cdot \hat{r}\right)+\left(\vec{m}_{i} \cdot \hat{r}\right)\left(\vec{m}_{j} \cdot \widehat{\theta}\right)\right]}{r_{i j}{ }^{4}}
$$


where $\mu_{0}$ is the vacuum magnetic permeability, $\mu_{c r}$ is the relative magnetic permeability of the continuous medium, $\vec{m}_{i}$ and $\vec{m}_{j}$ are the magnetic moments of the particles $i$ and $j$, and $\hat{r}$ and $\hat{\theta}$ are the radial and polar angle unit vectors, respectively.

Under the linear magnetization approximation, the magnetic moments can be expressed as a function of the magnetic field strength [18]: $\vec{m}_{i}=4 \pi \mu_{0} \mu_{c r} a^{3} \beta \vec{H}$. Here, $\beta$ is the so-called contrast factor that depends on the difference between the magnetic permeabilities of the particles and the continuous medium, $\beta=\left(\mu_{p r}-\mu_{c r}\right) /\left(\mu_{p r}+2 \mu_{c r}\right)$ where $\mu_{p r}$ is the relative magnetic permeability of the particles, and $\vec{H}$ is the magnetic field strength. The magnetic field strength was calculated using the local field approximation, $\vec{H}=\vec{H}_{l o c}$, which at the center position of the particle $i$ can be expressed as follows: [19]

$$
\begin{aligned}
\vec{H}_{l o c, i} & =\vec{H}_{0}+\sum_{j \neq i}^{N} \vec{H}_{m_{j}} \\
\vec{H}_{m_{j}} & =\frac{3\left(\vec{m}_{j} \cdot \hat{r}\right) \hat{r}-\vec{m}_{j}}{4 \pi \mu_{0} \mu_{c r} r_{i j}{ }^{3}}
\end{aligned}
$$

where $\vec{H}_{0}$ is the external magnetic field applied in the $z$-direction, $\hat{r}=\vec{r}_{i j} / r_{i j}$ is the unit vector and $\vec{H}_{m_{j}}$ is the magnetic field produced by the magnetic dipole, $\vec{m}_{j}$, at the center of the particle $i$. Owing to the mutual magnetization effects, the magnitudes of the local magnetic field and the magnetic moments of the particles must be solved in a selfconsistent manner. Although the magnetic interactions are long-ranged, a cutoff distance of $r_{c, \text { mag }}=l(t)$ was used in calculating the magnetic interactions in order to reduce the total simulation time. Different cutoff distances were compared in order to ensure that the particular cutoff radius used in simulations is large enough to obtain sensible results. The external magnetic field used in all the simulations was $H_{0}=$ $177 \mathrm{kA} / \mathrm{m}$ and the contrast factor for this magnetic field strength was estimated from the magnetization curves of typical MR fluids, $\beta=0.706$ [5].

The fluid-particle interaction was modeled by the Stokes' law. According to this, the drag force, $\vec{F}_{i}^{\text {hyd }}$, exerted by the fluid on the particle $i$, is given by the expression: $\vec{F}_{i}^{\text {hyd }}=-6 \pi \eta_{c} a\left(\frac{d \vec{r}_{i}}{d t}-\vec{u}_{i}^{\infty}\right)$, where $\eta_{c}$ is the continuous medium viscosity and $\vec{u}_{i}^{\infty}$ is 
the laminar viscous fluid velocity at the center of the particle $i$ (in the absence of particles). In the squeeze flow mode, under no-slip boundary conditions and creeping flow approximation, the velocity of the fluid is given by [20]:

$$
\vec{u}_{i}^{\infty}=\frac{3 v}{4} \frac{\rho_{i}}{h}\left(1-\frac{4 z_{i}^{2}}{h^{2}}\right) \hat{\rho}+\frac{v}{2} \frac{z_{i}}{h}\left(3-\frac{4 z_{i}^{2}}{h^{2}}\right) \hat{z}
$$

where, $\rho_{i}$ and $z_{i}$ stand for the cylindrical coordinates of the particle $i$, and $\hat{\rho}$ and $\hat{z}$ are the corresponding unit vectors. Equation 4 implies that the squeeze flow drives the particles in the central simulation box move outwards along the radial directions. The lateral dimensions of the box increase during compression maintaining the constant volume. This implies that the deformation of the box is not exactly in the same geometric way as the motion of the fluid, although the average velocity is the same at the boundaries. Therefore, the squeeze flow or hydrodynamic drag will not result in drift of particles out of the box. However, the other forces still allow them to leave the box from one side and come back from the opposite side. We used this approximation because of the convenient implementation of PBCs which could be very hard, if possible at all, to implement using cylindrical deforming boxes.

Short-range repulsive forces of exponential form, $\vec{F}_{i j}{ }^{\text {rep }}$ and $\vec{F}_{i}^{\text {wall }}$, were used to avoid overlap between the particles and between the particles and the walls, respectively,

$$
\begin{aligned}
& \vec{F}_{i j}^{\text {rep }}=-F_{0} \exp \left[-k\left(r_{i j}-2 a\right)\right] \hat{r} \\
& \vec{F}_{i}^{\text {wall }}=F_{0} \exp \left[-k\left(d_{i}-a\right)\right] \hat{n}
\end{aligned}
$$

Here, $F_{0}$ is defined as half the magnetostatic force between two magnetic dipoles in a head-to-tail configuration aligned in the direction of the external magnetic field (neglecting local field corrections): $F_{0}=\frac{3}{4} \pi \mu_{0} \mu_{c r} \beta^{2} H_{0}{ }^{2} a^{2} . k$ is the stiffness constant of the repulsive force and was chosen to be $k=50 / a . d_{i}$ is the distance between the center of the particle to the surface of a horizontal wall $\left(d_{i}=h / 2-z_{i}\right.$ for the upper wall and $d_{i}=h / 2+z_{i}$ for the lower wall) and $\hat{n}$ is the unit vector normal to that particular wall. Two cutoff radii were applied in calculating the repulsive forces 
between the particles and between the particles and the walls, i. e., $r_{c, r e p}=4 a$ and $r_{c, \text { wall }}=2 a$, respectively.

The motion of particle $i$ is governed by the Langevin equation:

$$
6 \pi \eta_{c} a \frac{d \vec{r}_{i}}{d t}=6 \pi \eta_{c} a \vec{u}_{i}^{\infty}+\vec{F}_{i}+\vec{f}_{B}(t)
$$

where $\vec{F}_{i}$ is the total force exerted on the particle $i$, and $\vec{f}_{B}(t)$ is a random force introduced to mimic the Brownian motion of the particle. The random force was calculated as $f_{B} \propto \sqrt{6 \pi \eta_{c} a k_{B} T / \Delta t}$, where $k_{B}$ is the Boltzmann constant, $T$ is the temperature and $\Delta t$ is the time variation. In this work we focus on isothermal conditions; the temperature was fixed at $T=298 \mathrm{~K}$. We used the following scales for the length, force and time: $l_{s}=2 a, F_{s}=F_{0}$ and $t_{s}=12 \pi \eta_{c} a^{2} / F_{0}$ in order to work in dimensionless units. Equation 7 was solved in this work using the Euler algorithm and the time variation was calculated at every step taking into account that the maximum displacement of a particle in any direction is $\Delta x, \Delta y, \Delta z=0.1 a$. The maximum of the time step was imposed to be $10^{-3}$ in dimensionless unit and the approaching velocity of the plates was set at $v^{*}=10^{-2}$.

In this work, the PBCs used are an extension of the Kraynik-Reinelt boundary conditions from planar extensional flows to biaxial extensional flows [21]. Dimensions of the box are changed simply reducing in the $z$-direction and expanding in the $x$ - and $y$ - directions to maintain the constant volume. Particles leaving the cuboid simulation box in the $x$ - and $y$ - directions are relocated by $x^{\prime}=x+$ floor $\left(\frac{x}{2 l}+\frac{1}{2}\right)$ and $y^{\prime}=y+$ floor $\left(\frac{y}{2 l}+\frac{1}{2}\right)$ where $x^{\prime}$ and $y^{\prime}$ are the coordinates after the relocation and the function floor $(x)$ returns the largest integer smaller than or equal to $x$. In the z-direction, no PBCs were applied but the effective wall repulsion.

The normal stress in the suspension was estimated as follows:

$$
\tau_{z z}=-\frac{1}{V} \sum_{i<j}^{N} z_{i j} F_{z, i j}
$$


where $z_{i j}$ is the difference between the z-coordinates of particles $i$ and $j$, and $F_{z, i j}$ is the $z$-component of the total pair-wise force between these two particles. However, a more easily accessible experimental observable is the normal force instead of the normal stress. The normal force was calculated in this work using two different approaches. On the one hand, the normal force acting on the upper plate was computed by multiplying the normal stress with the surface area of the plate $\left(l^{2}\right)$. Another approach is to calculate the normal force by differentiating the magnetic energy with respect to the gap height similarly to de Vicente et al. [5]. It is important to remark here that fluctuations in the energy due to the limited number of particles make it difficult to carry out the differentiation directly [22]. Instead we first fitted the simulation data on magnetic energy to a fourth-order polynomial and then differentiated the polynomial expression.

Three different kinds of simulations were carried out. In the first set of simulations, we compressed single-particle-width chains (having different number of particles) placed at the center of the simulation box. In the second set of simulations, the effect of increasing local concentration on the normal force, due to the sticky boundary conditions, was tested using systems consisting of single-particle-width spanning chains that are arranged either randomly or in a lattice pattern on the $x-y$ plane. Finally, in the third set of simulations, we compressed suspensions of magnetizable particles that were randomly distributed in the box at the initial time. The methodology consisted of two differentiated steps. First, particles inside the box were subjected to a suddenly applied magnetic field for structuration. Once the system reached a stationary state, in a second step, a squeeze flow field was superimposed in the presence of the magnetic field. In both steps, the magnetic energy, normal stress and average number of nearest neighbors per particle were monitored as a function of time. In the second step the normal force acting on the compressing plates (see Figure 1) was also calculated. Unless explicitly stated, the local field approximation in Equation 2 was used to calculate the fieldinduced dipole moments and so the normal stress.

\section{SINGLE-PARTICLE-WIDTH CHAINS}

The first set of simulations studied single-particle-width chains. These chains constitute the simplest structures possible and a first approximation to the squeeze flow behavior of real suspensions. In the simulation box, a preassembled single chain was initially aligned in the $z$-direction in a head-to-tail configuration spanning the entire gap 
between the two plates. Hence, the number of particles in the simulation box was proportional to the initial gap height according to $N=h_{0} / 2 a$. Tests were carried out for different initial gap heights and correspondingly different number of particles $(N \in[30,100])$. The particle loading $(\phi \in[0.01,0.20])$ was controlled by setting different initial lateral side lengths of the box. In all simulations the final gap height was fixed at $h_{f}=40 a$.

Figure 2a depicts the dimensionless normal stress $\tau_{z z}{ }^{*}$ as a function of $\frac{h}{h_{0}}=1-$ $\varepsilon$, where $\varepsilon=\left(h_{0}-h\right) / h_{0}$ is the compressive strain, at different particle loadings for chains of different lengths. The use of $1-\varepsilon$ is important in order to obtain dimensionless equations and split the compressive dependence on the other parameters involved in the squeeze behavior of MR fluids. The normal stress seems to be independent of the number of particles in the chain $(N)$ and therefore the initial gap height $\left(h_{0}\right)$. The stress remains essentially constant upon compression for the lowest particle concentrations, although some jumps are observed at the highest concentration (20 vol\%). The snapshots of these simulation systems could be used to interpret the stress variation under compression. For example, Figure 3 shows that a chain is broken during the compression test. The rupture happens at the extremes of the chain rather than in the middle. Jumps in the $\tau_{z z}{ }^{*}$ data can be related to the single-to-double chain transitions, especially in the most dilute case where the interaction with other chains is minimal. However, for the highest concentration, $\phi=0.20$, the stress decreases upon compression until $1-\varepsilon \approx 0.4$, and then jumps back to higher values at smaller gap height, reflecting a structural rearrangement from initial spanning single-particle-width chains to broken but not spanning chains attached separately to the two walls, and then to thicker column structures. Such non-monotonic trend was not observed in experiments and can be explained by the fact that single-particle-width chains do not actually exist at high particle concentrations. One also needs to bear in mind that owing to the PBCs magnetic interactions with neighboring image chains can affect the stress of the chain in the central box. The results in Figure 2a suggest that there is a critical concentration at approximately $\phi=0.10$ above which the hypothesis of singleparticle-width chains is not valid. This finding is in good agreement with previous reports in the recent literature involving both experiments and shearing flows [14,23-24] 
and also with the results obtained from MR fluid simulations with random initial configurations shown in Section V.

Inspection of Figure 2a suggests that the dimensionless normal stress is linearly dependent on the particle concentration as expected because the single chain configuration. This result is in good agreement with a recently proposed micromechanical model for squeeze flow [14]. According to this model, the yield compressive stress, obtained in the zero-strain limit, can be written as follows: $\tau_{Y C}=$ $\frac{27}{32} \phi \mu_{0} \mu_{c r} \beta_{a}{ }^{2} H^{2}$. Here, $\beta_{a}$ is the contrast factor of the aggregates, $H=(1-$ $\beta \zeta(3) / 2)^{-1} H_{0}$ is the local field of an infinite chain, $\beta$ is the contrast factor of the particles and $\zeta$ is the Riemann Zeta function. Figure $2 b$ shows the ratio between the simulation results on the dimensionless normal stress, $\tau_{z z}{ }^{*}$, and the dimensionless yield compressive stress predicted by the referred micromechanical model, $\tau_{Y C}{ }^{*}=\frac{9}{2 \pi} \phi \frac{\beta_{a}{ }^{2} H^{2}}{\beta^{2} H_{0}{ }^{2}}$. As shown in Figure $2 \mathrm{~b}$, the $\tau_{z z}{ }^{*}$ data scale reasonably well with the theoretical predictions. The variance of all the data points collected from different concentrations and chain sizes in the zero-strain limit $(\varepsilon=0)$ is below $25 \%$, suggesting that the rescaled stress data reasonably collapse onto a master curve. Despite the good scaling with the particle concentration and magnetic field strength, there is still a difference of about a factor of 3 between simulation results and micromechanical predictions. Obviously, discrepancy between the simulation and theoretical results becomes more apparent for the largest concentrations as a result of the inter-aggregate interactions. These interactions are not taken into account in the micromechanical model.

Next, the normal force $F_{z z}$ acting on the confining plates was calculated from $\tau_{z z}$ by simply multiplying with the surface area $S$ (Figure 4):

$$
F_{z z}=\tau_{z z} S=\tau_{z z} \frac{V}{h}=-\frac{1}{h} \sum_{i<j}^{N} z_{i j} F_{z, i j}
$$

For constant volume $V$, the normal force is simply proportional to the normal stress divided by the gap height. Since $\tau_{z z}$ remains essentially constant during the compression, the dimensionless normal force $F_{z z}^{*}$ should scale linearly with $1 /(1-\varepsilon)$ $\left(=h_{0} / h\right)$. This scaling behavior is supported by the dimensionless normal force data in 
Figure 4 where the results obtained at different particle concentrations $\phi$ collapse onto a master curve. This collapse of the $F_{z Z}^{*}$ data is to be expected, because the force scale factor $F_{0}$ can be rewritten to show its linear dependence on the particle concentration $\phi$ as the particle diameter can be expressed as $a^{3}=3 V \phi / 4 N \pi$. For gap-spanning singlewidth chains, the number of particles is simply $N=h_{0} / 2 a$ and $a^{2}=3 V \phi / 4 h_{0} \pi$. Substituting this in the force scale factor, a linear dependence on the particle concentration is found $F_{0}=\frac{9}{8} \mu_{0} \mu_{c r} \beta^{2} H_{0}^{2} \phi \frac{V}{h_{0}}$. It is also important to remark that this normal force scale factor is proportional to the yield force obtained in the micromechanical model proposed by Ruiz-López et al. [14].

Although the simulation results on the normal stress and normal force follow the same dependence on the particle concentration and the magnetic field squared as both the yield compressive stress and yield normal force predicted by theoretical approaches, their dependence on the compressive strain is different from the model prediction. Simulations revealed a scaling of the normal force with $(1-\varepsilon)^{-1}$, while the micromechanical model predicts a scaling of $(1-\varepsilon)^{-2}[14]$. This difference can be explained by the results in Figure 2 that in the simulations explored so far the normal stress remains essentially unaltered during compression, mainly because of the breakage of the single-particle-width chains in the process. However, the micromechanical model has taken into account the thicker chain or column structures, just as observed in experiments.

\section{RANDOM AND LATTICE ARRANGEMENTS OF SINGLE-WIDTH- PARTICLE CHAINS}

In a typical experiment, if the no-slip conditions are very strong and the compression velocity is sufficiently slow, it is reasonable to think that the initial chainlike structures remain stuck in their initial positions (within $r_{0}$ ) leading to an increase in the local concentration of particles in that region. The normal force can increase upon compression because of two reasons: i) by an increase in the local concentration of particles, and ii) by changes in the aspect ratio of the field-induced structures under squeeze. To separate these two contributions, we carried out a second set of simulations which were focused on evaluating the effect of local concentration in the absence of chain thickness variation. We calculated the normal stress for different preassembled 
structures at different gap heights. In this case, the total number of particles in the simulation box was fixed to $N=3600$. The magnetic dipole moments of the particles and correspondingly the normal stress were calculated using either only the external field strength $H_{0}$ or the local field approximation $H_{l o c}$ as given in Equation 2. Although the lateral dimensions of the constant-volume simulation box increase during the squeeze process, we assume that the particles do not move out of the region defined by the original lateral dimensions of the box. As a result, the local concentration of particles inside this region was increased by gradually reducing the gap between the two plates, although the overall particle concentration in the simulation box remains to be the same. The overall and also the initial local concentration of particles was taken to be 5 vol\% while the final local concentration increased up to 22.6 vol\%. At each given gap height or local concentration, the particles are re-arranged into single-particle-width chains that span the gap and are distributed in the x-y plane either in cubic or hexagonal lattices, or randomly. Thus, for the fixed number of particles, the number of chains in the region with fixed lateral dimensions was increased when the gap diminished, and the mean lateral distance between them was reduced.

Simulation results on the normal stress $\tau_{z z}{ }^{*}$ obtained both with and without the application of PBCs are presented in Figure 5. The first observation is that the particular arrangement of the chains, either in a lattice or in random distribution, has negligible effect on the normal stress, as reflected in the nearly identical results obtained from the different chain location arrangements. The results are also insensitive to the boundary conditions in the lateral directions. The second observation is that $\tau_{z z}{ }^{*}$ strongly depends on the field approximation used for calculating the magnetic dipole moments. At low compressive strains, the normal stress calculated using the local field correction (Equation 2) is much higher than that using the external field, in good agreement with simulation results in literature $[19,25]$. The third observation is that the simulated normal stress decreases during compression, which is more obvious when using the local field corrections. This is in clear contrast to experimental results reported in literature for concentrated MR fluids [7-9] and theoretical predictions [14]. It suggests that for concentrated MR fluids, the use of the dipole-dipole approximation for the magnetic forces, the simple Stokes' law for the hydrodynamic interactions as well as the single-particle-width chains in these simulations is not enough to quantitatively, and sometimes even qualitatively, predict their magnetorheological behavior. Other effects 
must be considered. For example, the lubrication and friction forces have been used in high-solid-content colloidal dispersions as a cause of the shear thickening [26]. They might be also important in the squeeze flow of MR suspensions.

\section{MAGNETORHEOLOGICAL FLUIDS WITH RANDOM INITIAL CONFIGURATIONS}

\section{A. Field-induced structuration in quiescent state}

The initial configurations of the MR fluids studied in this section consist of magnetic particles randomly dispersed in the carrier liquid. Upon application of the external field, the particles aggregate to form clusters. To characterize the field-driven structuration process, the magnetic interaction energy per particle, $U^{*} / N$, the normal stress, $\tau_{z Z}^{*}$, and the average number of nearest neighbors per particle (within a center-tocenter distance of $2.2 a$ ), $N_{\text {near }}$, are shown in Figure 6 as a function of time for the MR fluids with particle loadings $\phi \in[0.05,0.30]$. In each case the simulation results were averaged over at least 5 independent simulation runs.

After a dimensionless time of $t^{*} \approx 500$, the magnetic energy is saturated (Figure 6a), which suggests that the structure achieves a stationary state. The saturation times are even shorter for the most concentrated suspensions because of the shorter mean free paths for the particles to move into their stable aggregation positions. Simultaneously, the normal stress and average number of nearest neighbors also reach constant values (Figure $6 \mathrm{~b}$ and Figure 6c, respectively). With the increase of the particle concentration, larger clusters or thicker column structures are formed, leading to the increase of the average number of nearest neighbors per particle. As a consequence, the absolute value of the (attractive) magnetic energy per particle decreases with $\phi$, because the particles cannot find their most stable (head-to-tail) position in the more compact structures generated at higher particle volume fractions. But the normal stress of the system at the stationary state prior to compression still increases upon increasing $\phi$. It is important to remark that the average number of nearest neighbours per particle is larger than 2 , so the ideal model of single-particle-width chains is not suitable to explain these results even for a MR fluid with $\phi=0.10$. In the previous sections, the single-width-particle chains have been used in simulations in order to better understand the configuration of chains in squeeze. However, this situation is not real, because the particles usually arrange into thicker aggregates or column structures that are formed by multiple single-width- 
particle chains. The validity of the simulations using single-width-particle chains is limited to dilute suspensions.

\section{B. Squeeze flow}

Squeeze flow simulations were carried out using the stationary-state structures obtained above as initial configurations. In Figure 7, the simulation results on $U^{*} / N$, $\tau_{z z}^{*}$ and $N_{\text {near }}$ are shown as functions of $1-\varepsilon$ during the compression of the MR fluids. It can be seen in Figure $7 \mathrm{a}$ that for a given particle volume concentration the absolute value of the (attractive) magnetic energy per particle decreases as the strain increases. Although the curves for the dimensionless magnetic energy per particle as shown in Figure 7a seem to be just shifted with one another and their derivatives seem to be similar (see also Figure 9), the total magnetic energy of the system is proportional to multiplication of the concentration and these data, $U=\frac{9}{8} \mu_{0} \mu_{c r} \beta^{2} H_{0}^{2} V \phi \frac{U^{*}}{N}$. Thus, the total magnetic energy grows more sharply at higher particle volume fractions. This is expected due to the fact that the derivative of the magnetic energy gives the normal force and the latter is well known to increase when increasing particle concentration [79]. Figure $7 \mathrm{~b}$ shows that the normal stress increases with increasing $\phi$, similar to the $\tau_{z z}^{*}$ results obtained from the single-particle-width chain simulations (see Figure 2a). Also, the normal stress decreases during compression. This fact can be explained in terms of the decrease of the normal stress obtained when increasing the local concentration (see Figure 5). Thus, the inter-chain repulsive interaction becomes more important during compression leading to a reduction of the normal stress.

In Figure 7c we show the average number of nearest neighbors per particle, $N_{\text {near }}$. For $\phi=0.05$ this number increases upon compression as expected, because compression can assist the formation of thicker aggregates. However, at higher particle concentrations, the value of $N_{\text {near }}$ initially increases with the decrease of the gap height for relatively large $h^{*}$ values, but then decreases. This can be explained by the fact that thicker aggregates are formed in the initial stages of compression, but after a certain compressive strain value, these structures break apart under the action of the viscous drag and so result in a decrease of $N_{\text {near }}$. In Figure 8, we present the peak values of the pair distribution functions of the particles, $g(r)$, as a function of the compressive strain $\varepsilon$ and the particle concentration $\phi$. Apart from the system with the lowest concentration 
$\phi=0.05$ where the peak value increases monotonically with $\varepsilon$, the peak heights of $g(r)$ in all other systems reach a maximum at certain compressive strain and then decrease, reflecting a reduction in $N_{\text {near }}$. In addition, the peak values in the systems with higher particle concentrations are smaller than those at lower $\phi$, indicating that the average number of nearest neigbhors per particle does not grow linearly with the particle concentration.

Figure 9 presents the simulation results on the normal forces, $F_{z Z}^{*}$, divided by the dimensionless yield normal force predicted by the micromechanical model [14], $F_{Y}{ }^{*}=\frac{9}{2 \pi} \phi \frac{\beta_{a}{ }^{2} H^{2}}{\beta^{2} H_{0}{ }^{2}} \frac{V^{*}}{h_{0}{ }^{*}}$. The normal force was calculated using two different approaches: i) by multiplying the normal stress with the plate surface area (Equation 9) and ii) by taking the derivate of the magnetic energy with respect to the gap height (see Equation 23 in [5]). Results generated by these two methods show very different compressive behavior. At the onset of the compression, the $F_{z Z}^{*}$ data calculated using Equation 9 are higher than those obtained by taking the derivative of the magnetic energy. However, the normal force obtained in the latter case increases more rapidly under compression, and so the two sets of data approach each other at high compressive strains. These differences could be partly explained by the numerical accuracy of the two approaches. It is worth to remark that the calculations were carried out by including only the magnetic energy, since the short-range exponential repulsive forces are more susceptible to particle position perturbations and the results are not sensible. The derivative of the energy is more sensitive to the numerical errors in the simulations. Nevertheless, as shown in Figure 9, the normal force obtained by the energy differentiation approach shows a change of slope during compression. The exponent, $b$, of the normal force with the gap, $F \sim(1-\varepsilon)^{-b} \sim h^{-b}$ is larger than 2 for small compressions $\varepsilon \in[0,0.2]$ and smaller than 2 for large compressive strains $\varepsilon>0.5$. It seems to approach a value of 2 for intermediate compressive strains $\varepsilon \in[0.2,0.5]$, which agrees with the predictions of the micromechanical model and also the experimental data [14]. But the $F_{z Z}^{*}$ results calculated by multiplying normal stress with plate surface area show a much weaker power law dependence on $1-\varepsilon$. At the highest particle concentrations, the calculated normal force even decreases under compression. The discrepancy between the simulation and theoretical/experimental results could be related to the strong decrease of the normal stress $\tau_{z z}^{*}$ with $\varepsilon$, see Figure $7 \mathrm{~b}$. As 
elucidated in Figure 5, the decrease of $\tau_{z z}^{*}$ with the increase of the local particle concentration upon compression arises from the net repulsive forces among chains. Thus, the mechanisms underlining the squeeze flow behaviour of MR fluids with high concentrations cannot be obtained in terms of only dipolar and repulsive forces between particles. Other interactions should be included.

The zero-strain values of the normal stress in Figure $7 \mathrm{~b}$ correspond to the socalled yield compressive stress $\tau_{Y C}^{*}$. Figure 10 depicts the $\tau_{Y C}^{*}$ results obtained from the simulations using the single-particle-width chain model systems (squares) and from MR suspensions with random initial configurations (circles) as well as from experiments (triangles) as a function of the particle concentration [14]. The micromechanical model predictions (solid line) are also included for completeness [14]. The yield compressive stresses given by the single-particle-width chain simulations are higher than the theoretical predictions, regardless of particle concentration. When compared with the simulation results from MR suspensions, a good agreement is achieved at the lowest concentrations, but deviations occur at higher $\phi$ values due to the inter-chain repulsive force. Overall, the single-width-chain simulation results and theoretical predictions show a linear dependence of the yield compressive stress on the particle concentration, and the simulation data from the MR suspensions demonstrate a sub-linear behavior. However, the experimental data exhibit a stronger dependence on $\phi$ above $\phi=0.05$, which is in good agreement with other experimental works reported in the literature [79]. It indicates that further improvements on the simulation model are still needed for providing quantitative predictions of the squeeze flow behavior of MR fluids as for instance the inclusion of lubrication and frictional forces [26].

\section{CONCLUSIONS}

Brownian dynamics simulations of model MR fluids were carried out under squeeze flow for different types of initial structures: one single-particle-width chain per simulation box, systems consisting of gap-spanning single-particle-width chains arranged randomly and in regular lattices and suspensions of randomly distributed particles at different concentrations. Simulations using randomly and regularly arranged single chains demonstrated that the use of the local field approximation for dealing with the magnetic interactions can improve the normal stress and normal force results over those obtained by simply using the external magnetic field strength not only 
quantitatively, but also sometimes qualitatively (e. g., at relatively small plate-plate separations). Therefore, simulation results in all other cases were obtained using the local field approximation.

Simulation results obtained from the systems with one single-particle-width chain per box showed that the normal force and so the yield compressive stress scale linearly with the particle volume fraction and the magnetic field strength squared, which is in agreement with the micromechanical model developed by Ruiz-López et al. [14] and experiments reported in literature. But the dependence of the normal force (and the compressive stress) on the compressive strain found in these simulations differ from those of the micromechanical model predictions and the experimental data. A close inspection of the snapshots of the simulation systems revealed that under compression the originally gap-spanning single chains broke into short non-spanning chains or clusters, which leads to a nearly unaltered normal stress. As a result, the normal force shows weaker dependence (power of -1) on the gap height in comparison with the power of -2 scaling found in experiments and micromechanical model calculation where the particles can form thicker aggregates.

Simulation results obtained from the pre-arranged configurations consisting of single-particle-width chains showed the effect of the local concentration. While the normal stress calculated using one single-particle-width chain per simulation box remains practically constant during compression, the existence of neighboring chains in the pre-arranged systems leads to an important decrease in the normal stress, because of the increase of the local particle concentration and the repulsive inter-chain forces. This effect is also reflected in the simulations of MR suspensions with random initial configurations where the normal force calculated as a multiplication of the normal stress and the plate surface area shows a much weaker dependence on the compressive strain than that found in experiments or in the micromechanical model. This fact suggests that other inter-particle forces, such as the lubrication and friction forces, must be included in the simulation model to obtain more realistic results.

It is noted that the yield compressive stress obtained in the single-particle-width chain simulations shows a linear particle-loading dependence, in good agreement with the micromechanical model. On the contrary, in experiments, deviations from linearity 
appear at higher concentrations. Although this discrepancy between micromechanical models and experimental results has been usually explained by the existence of interactions between chain-like structures (e.g. $[12,14]$ ), simulations of MR suspensions with initially randomly distributed particles still showed the linear or sub-linear dependence of the yield compressive stress on the particle concentration. Further understanding of the experimental observations are still needed.

\section{ACKNOWLEDGEMENTS}

This work was supported by MAT 2016-78778-R, MAT 2013-44429-R and PCIN2015-051 projects (MINECO and FEDER, Spain; H2020, UE) and by Junta de Andalucía P11-FQM-7074 project (Spain). J.A.R.-L. acknowledges the financial support by the "Ministerio de Educación: Becas del Programa de Formación del Profesorado Universitario (FPU)" (AP2010-2144). 


\section{REFERENCES:}

[1] Bossis, G., O. Volkova, S. Lacis and A. Meunier, "Magnetorheology: Fluids, Structures and Rheology", in Ferrofluids, ed. S. Odenbach (Springer, Bremen, Germany, 2002), p. 202.

[2] de Vicente, J., D. J. Klingenberg and R. Hidalgo-Álvarez, "Magnetorheological fluids: a review," Soft Matter 7, 3701-3710 (2011a).

[3] Wereley, N. M., Magnetorheology: Advances and Applications (RCS Smart Materials, London, 2013).

[4] Havelka, K. O., and J. W. Pialet, "Electrorheological technology: The future is now," CHEMTECH 36, 36-45 (1996).

[5] de Vicente, J., J. A. Ruiz-Lopez, E. Adnablo-Reyes, J. P. Segovia-Gutierrez and R. Hidalgo-Alvarez, "Squeeze flow magnetorheology," J. Rheol. 55(4), 753-779 (2011b).

[6] Galindo-Rosales, F. J., J. P. Segovia-Gutierrez, F. T. Pinho, M. A. Alves and J. de Vicente, "Extensional Rheometry of Magnetic Dispersions," J. Rheol. 59(1), 193-209 (2015).

[7] Ruiz-Lopez, J. A., R. Hidalgo-Alvarez and J. de Vicente, "On the validity of continuous media theory for plastic materials in magnetorheological fluids under compression," Rheol. Acta 51(7), 595-602 (2012).

[8] Guo, C. Y., X. L. Gong, S. H. Xuan, L. J. Qin and Q. F. Yan, "Compression behaviors of magnetorheological fluids under nonuniform magnetic field," Rheol. Acta 52(2), 165-176 (2013).

[9] Xu, Y., T. Liu and S. Xuan, "Squeeze flow behaviors of magnetorheological plastomers under constant volume," J. Rheol. 58(3), 659-679 (2014).

[10] Tang, X., X. Zhang, R. Tao and Y. Rong, "Structure-enhanced yield stress of magnetorheological fluids," J. Appl. Phys. 87(5), 2634-2638 (2000).

[11] Becnel, A. C., S. G. Sherman, W. Hu and N. M. Wereley, "Squeeze strengthening of magnetorheological fluids using mixed mode operation," J. Appl. Phys. 117(17), 17C708 (2015).

[12] Zhang, X. Z., X. L. Gong, P. Q. Zhang and Q. M. Wang, "Study on the mechanism of the squeeze-strengthen effect in magnetorheological fluids," J. Appl. Phys. 96(4), 2359-2364 (2004). 
[13] Bigue, J. P. L., F. Charron and J.-S. Plante, "Understanding the super-strong behaviour of magnetorheological fluid in simultaneous squeeze-shear with the Peclet number," J. Intell. Mater. Sys. Struct. 26(14), 1844-1855 (2015).

[14] Ruiz-Lopez, J. A., R. Hidalgo-Alvarez and J. de Vicente, "A micromechanical model for magnetorheological fluids under slow compression," Rheol. Acta 55(3), 245256 (2016a).

[15] Lukkarinen, A., and K. Kaski, "Computational studies of compressed and sheared electrorheological fluid," J. Phys. D: Appl. Phys. 29, 2729-2732 (1996).

[16] Lukkarinen, A., and K. Kaski, "Simulation studies of compressed and sheared electrorheological suspensions," J. Appl. Phys. 83(3), 1717-1725 (1998).

[17] Kim, D. H., S.-H. Chu, K. H. Ahn and S. J. Lee, "Dynamic simulation of squeezing flow of ER fluids using parallel processing," Korea-Aust. Rheol. J. 11(3), 233-240 (1999).

[18] Ruiz-Lopez, J. A., R. Hidalgo-Alvarez and J. de Vicente, "Testing the mean magnetization approximation, dimensionless and scaling numbers in magnetorheology," Soft Matter 12(5), 1468-1476 (2016b).

[19] Wang, Z., Z. F. Lin and R. B. Tao, "Many-body effect in electrorheological responses," Int. J. Mod. Phys. B 10(10), 1153-1166 (1996).

[20] Engmann, J., C. Servais, and A. S. Burbidge, "Squeeze flow theory and applications to rheometry: A review," J. Non-Newtonian Fluid Mech. 132, 1-27 (2005).

[21] Baranyai, A. and Cummings, P. T., "Nonequilibrium molecular dynamic study of shear and shear-free flows in simple fluids,” J. Chem. Phys, 103, 10217 (1995).

[22] Ahnert, K., and M. Abel, "Numerical differentiation of experimental data: local versus global methods," Comput. Phys. Commun. 117(10), 764-774 (2007).

[23] Segovia-Gutierrez, J. P., C. Berli and J. de Vicente, "Nonlinear viscoelasticity and two-step yielding in magnetorheology: A colloidal gel approach to understand the effect of particle concentration," J. Rheol. 56(6), 1429-1448 (2012).

[24] Fernandez-Toledano, J. C., J. Rodriguez-Lopez, K. Shahrivar, R. Hidalgo-Alvarez, L. Elviera, F. M. de Espinosa and J. de Vicente, "Two-step yielding in magnetorheology," J. Rheol. 58(5), 1507-1534 (2014).

[25] Wang, Z., K. Shahrivar and J. de Vicente, "Creep and Recovery of Magnetorheological Fluids: Experiments and Simulations," J. Rheol. 58(6), 1725-1750 (2014). 
[26] Mari, R., R. Seto, J. F. Morris and M. M. Denn, "Shear thickening, frictionless and frictional rheologies in non-Brownian suspensions," J. Rheol. 58(6), 1693-1724 (2014). 


\section{FIGURES}

Figure 1.- Schematics of the simulation box and Cartesian coordinate system.

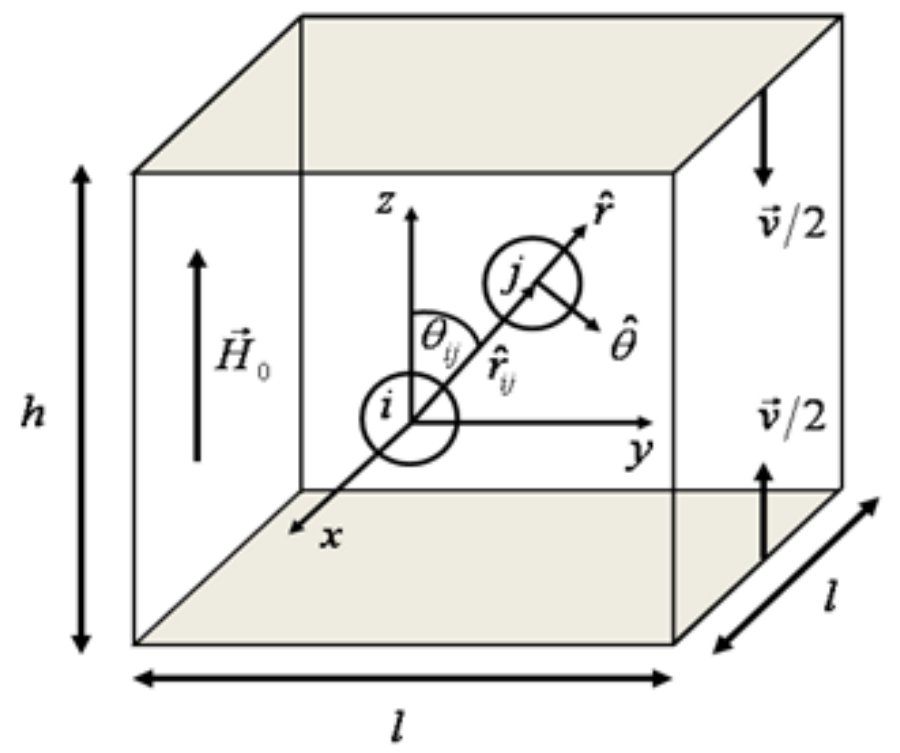


Figure 2.- a) Dimensionless normal stress $\tau_{z z}^{*}$ in single-particle-width chain simulations for chains of different lengths $(N \in[30,100])$ and particle loadings $(\phi \in[0.01,0.20])$ and b) dimensionless normal stress divided by the dimensionless yield compressive stress from the micromechanical model by Ruiz-López et al. [14]: $\tau_{Y C}{ }^{*}=\frac{9}{2 \pi} \phi \frac{\beta_{a}{ }^{2} H^{2}}{\beta^{2} H_{0}{ }^{2}}$ as a function of the dimensionless gap, $h / h_{0}=1-\varepsilon$. From bottom to top: solid symbols for $\phi=0.01$, open symbols for $\phi=0.05$, plus symbols for $\phi=0.10$ and cross symbols for $\phi=0.20$, respectively.
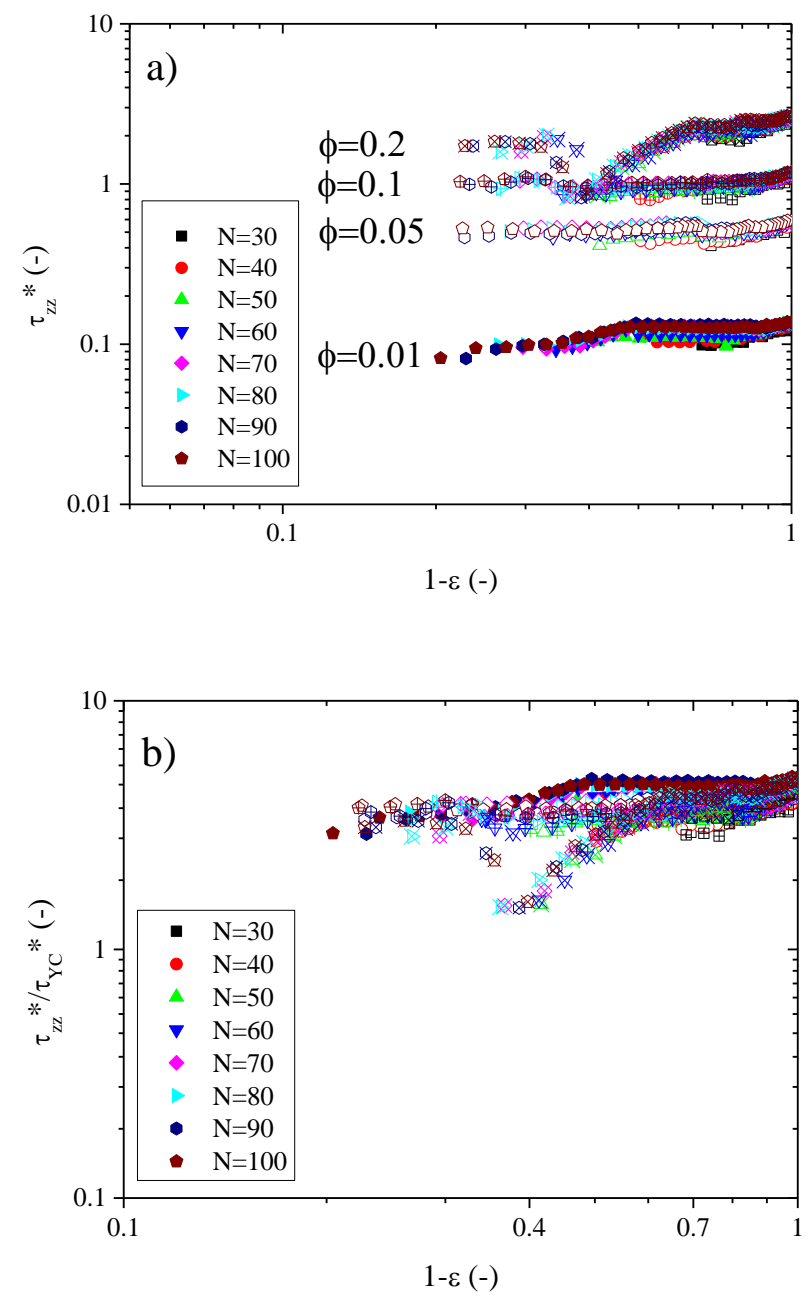
Figure 3.- Snapshots of a single-particle-width chain of length $N=140$ at particle loading $\phi=0.05$ under squeeze flow at different compression stages: a) $\varepsilon=0$; b) $\varepsilon=0.3$ and c) $\varepsilon=0.5$.

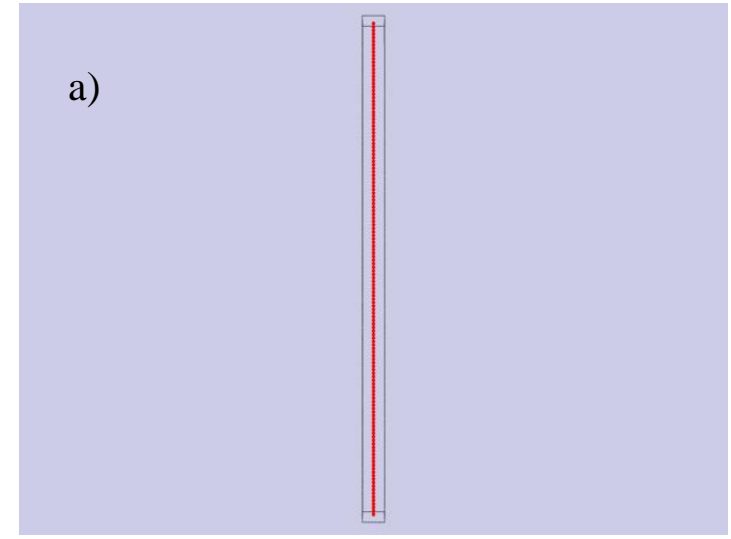

b)

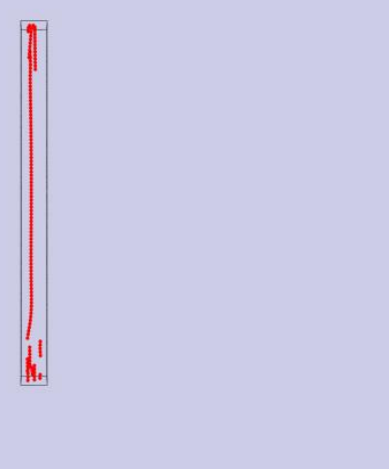

c)

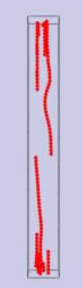


Figure 4.- Dimensionless normal force $F^{*}$ as a function of the dimensionless gap, $h / h_{0}=1-\varepsilon$, in single-particle-width chains simulations for chains of different lengths $(N \in[30,100])$ at different particle loadings $(\phi \in[0.01,0.20])$. The solid symbols are for systems with $\phi=0.01$, open symbols for $\phi=0.05$, plus symbols for $\phi=0.10$ and cross symbols for $\phi=0.20$.

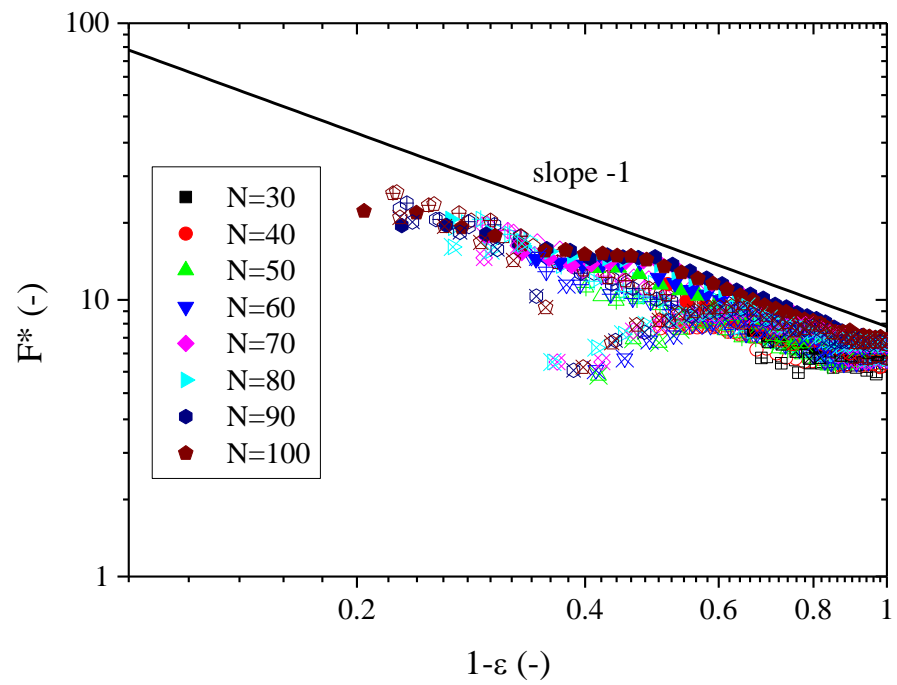


Figure 5.- Dimensionless normal stress $\tau_{z z}^{*}$ as a function of the dimensionless gap, $h / h_{0}=1-\varepsilon$, demonstrating the importance of particle concentration and local field correction in the squeeze flow. The results were obtained using either only the external field strength (triangles) or the local field approximation (Equation 2) (squares and circles). Solid symbols correspond to simulations using periodic boundary conditions (PBC) and open symbols correspond to simulations not using PBC

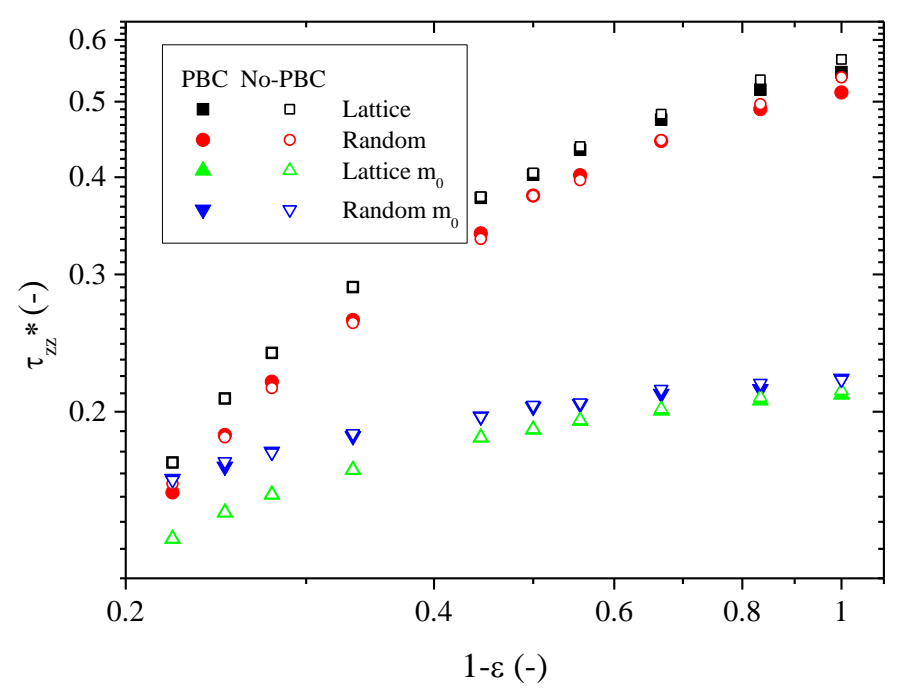


Figure 6.- Characterizing field-induced structuration process of MR fluids in quiescent state: a) magnetic energy, b) normal stress and c) average nearest neighbors per particle.
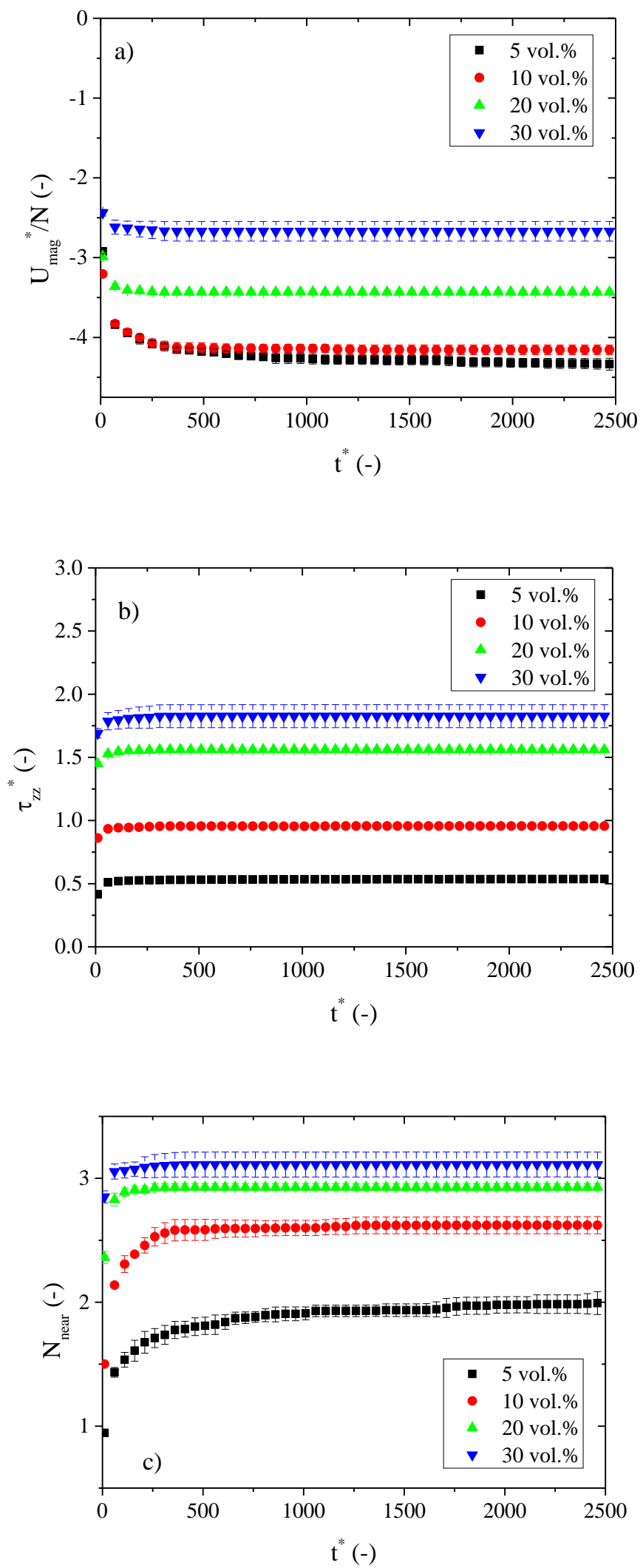
Figure 7.- Squeeze flow simulation results on a) magnetic energy per particle, b) normal stress and c) average number of nearest neighbours per particle of MR fluids with random initial configurations as functions of the dimensionless gap, $h / h_{0}=1-\varepsilon$.
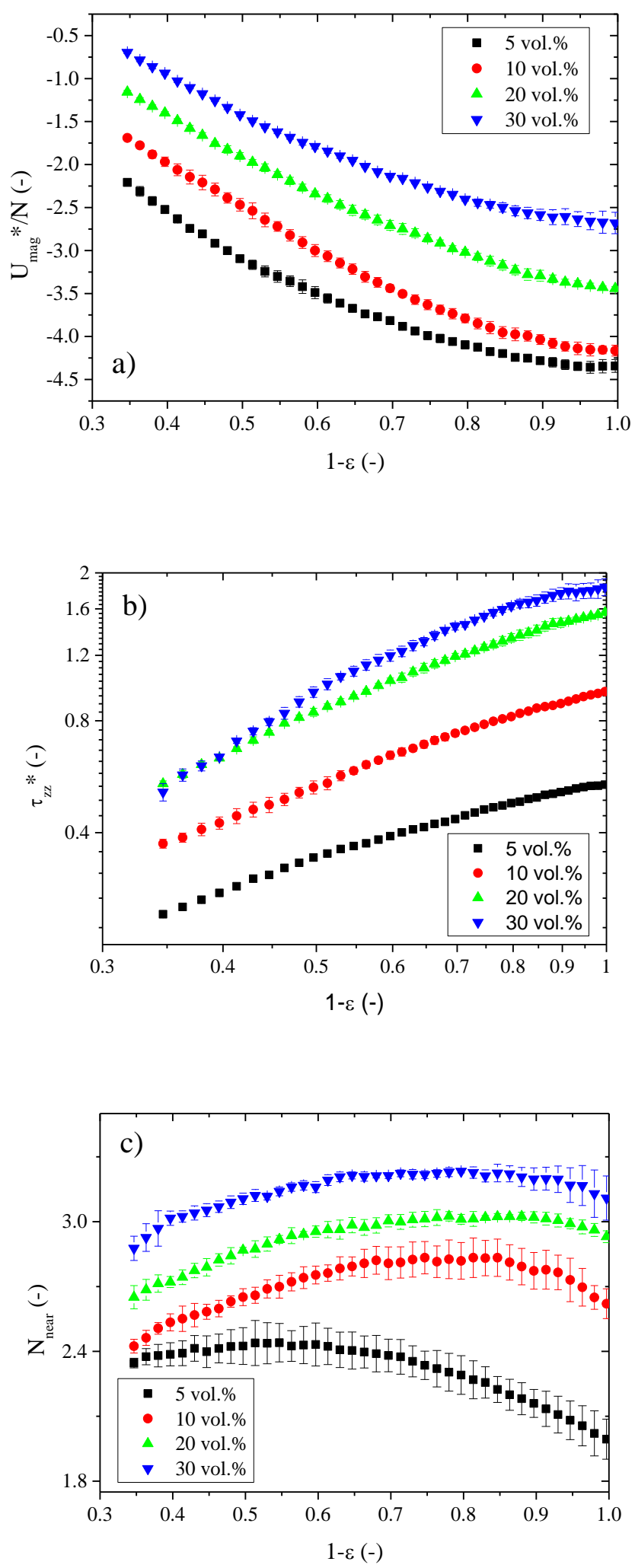
Figure 8.- Values of the highest peaks in the pair distribution functions of particles, $g(r)$, as a function of the dimensionless gap, $h / h_{0}=1-\varepsilon$, at different particle concentrations.

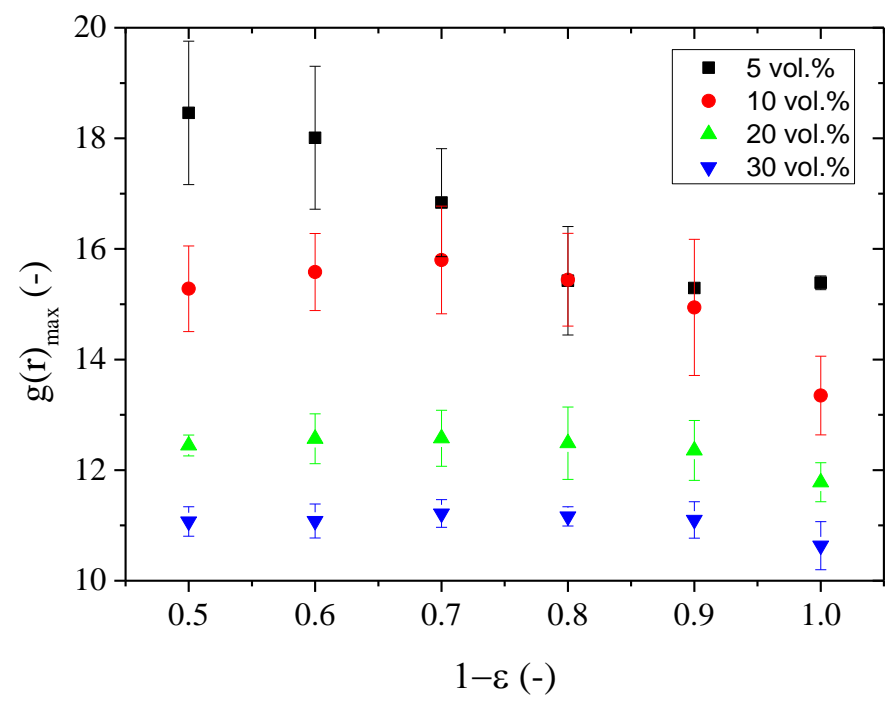


Figure 9.- Dimensionless normal force $F_{z Z}^{*} / F_{Y}^{*}$ as a function of the dimensionless gap, $h / h_{0}=1-\varepsilon$, for MR suspensions with random initial configurations calculated by i) the multiplication of the normal stress with the surface area using Equation 9 (solid symbols) and ii) the derivative of the magnetic energy with respect to the gap height using Equation 23 in [5] (open symbols). The dashed black line corresponds to the prediction of the micromechanical model for squeeze flow [14]: $F_{z Z}^{*} / F_{Y}^{*}=1 /(1-\varepsilon)^{2}$. The dimensionless yield normal force $F_{Y}^{*}$ is taken from that micromechanical model as $F_{Y}{ }^{*}=\frac{9}{2 \pi} \phi \frac{\beta_{a}^{2} H^{2}}{\beta^{2} H_{0}{ }^{2}} \frac{V^{*}}{h_{0}{ }^{*}}$.

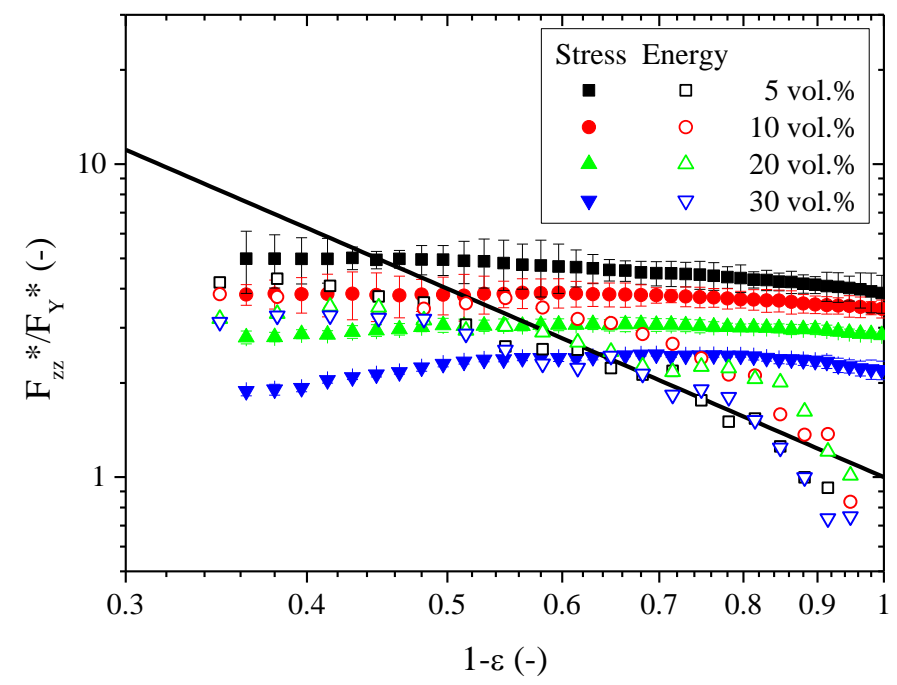


Figure 10.- Yield compressive stress $\tau_{Y C}^{*}$ obtained from simulations using the singleparticle-width chain systems (squares) and MR fluids with random initial configurations (circles) as well as from experiments [14] as a function of the particle concentration. Black line corresponds to the prediction of the micromechanical model for squeeze [14].

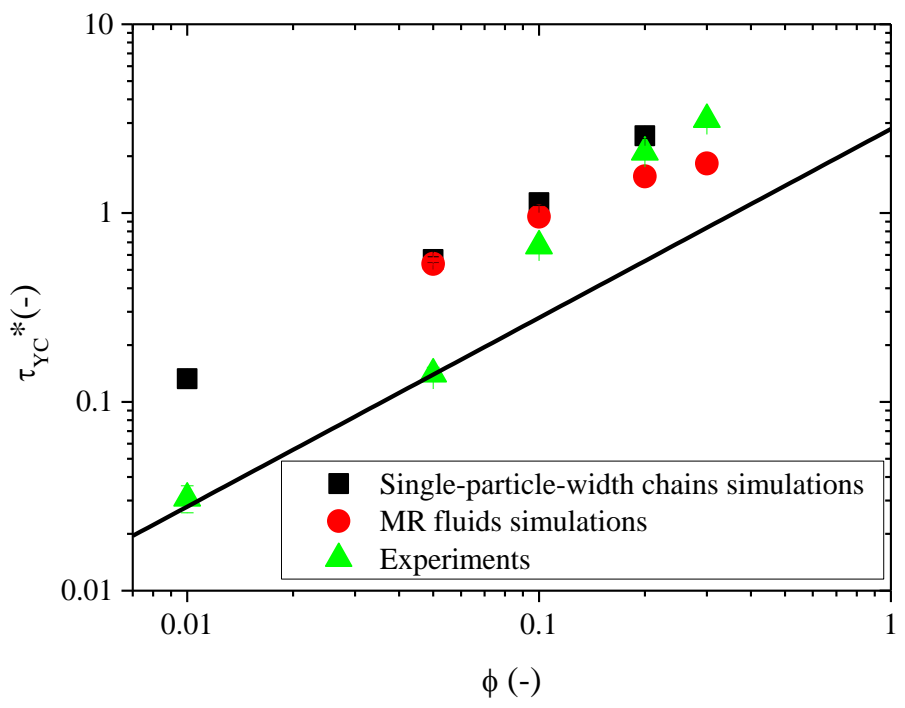

